\section{- EDUCATIONAL}

RESOURCE

Volume 9 Issue 32017

DOI: 10.21315/eimj2017.9.3.9

\section{ARTICLE INFO}

Submitted: 29-06-2017

Accepted: 16-07-2017

Online: 30-09-2017

\section{When Maximum Pain Control of Chronic Non- Malignant Condition Fails: Is There Anything Else To Do?}

\author{
Fahisham Taib', Jamilu Abdullahi Faruk², Ochaya Odong ${ }^{3}$, \\ Soumyadeep Biswa ${ }^{4}$, Bakht Jamal ${ }^{5}$, Meenal Mavinkurve ${ }^{6}$ \\ ${ }^{1}$ Department of Paediatrics, School of Medical Sciences, Universiti \\ Sains Malaysia, MALAYSIA \\ ${ }^{2}$ Paediatrics Department, Ahmadu Bello University, Zaria, Nigeria \\ ${ }^{3}$ Department of Paediatrics, International Hospital Kampala, \\ UGANDA \\ ${ }^{4}$ Paediatric Department, University Hospital of Wales, Cardiff, \\ WALES \\ ${ }^{5}$ Paediatric Department, University Hospital Kerry, Tralee, Co. Kerry, \\ IRELAND \\ ${ }^{6}$ Department of Paediatrics, Clinical School, International Medical \\ University, Seremban, Negeri Sembilan, MALAYSIA
}

To cite this article: Taib F, Abdullahi Faruk J, Odong O, Biswa S, Jamal B, Mavinkurve M. When maximum pain control of chronic non-malignant condition fails: is there anything else to do? Education in Medicine Journal. 2017;9(3): 83-87. https://doi.org/10.21315/eimj2017.9.3.9

To link to this article: https://doi.org/10.21315/eimj2017.9.3.9

\begin{abstract}
When patients are tethered with uncontrolled pain, health care practitioners usually resort to several modes of symptomatic relief for their patients. In palliative care the goal is identifying and managing the cause of pain in addition to addressing associated problems. To administer effective symptom control in patient with chronic non-cancer associated pain, healthcare professionals may need to consider alternative non-pharmacological modes of treatment such as psychological intervention. Pain perception can be influenced by non-organic factors such as emotional, psychosocial status of the patient and their religious and spiritual beliefs. Taking these factors into consideration are an essential part of treatment goals. The paper illustrates an ethical dilemma which has arisen in the aggressive management of pain and highlights the importance of multi-disciplinary involvement in patient care. Failure to consider these aspects of care can cause patient and parental anxiety, poor symptom control and could compromise doctor-patient relationship.
\end{abstract}

Keywords: Pain, Chronic pain, Non-malignant pain, Pain control, Psychology

CORRESPONDING AUTHOR Fahisham Taib, School of Medical Sciences, Universiti Sains Malaysia, 16010 Kubang Kerian, Kelantan, Malaysia | Email: fahisham@gmail.com 


\section{INTRODUCTION}

The World Health Organization (WHO) has coined pain as the 'fifth vital sign' and thus should be included in routine medical assessment of patients. Pain management should be prioritised in the clinical management of all acutely or chronically ill patients. If left untreated or inadequately addressed, pain can cause marked physiological changes and psychological distress to the patients. Pain management is regarded as a challenging standard for behavioural health care organisations to achieve due to its complexity and variability (1). Delivering individualised treatment, an important goal for chronic non-malignant pain management, poses a challenge to health professionals as it hinges on assessment of pain, additional clinical features of the underlying disease entity and consideration of patient's experiences. The WHO pain ladder is an accepted approach to the general pain management, but it lacks of approach to chronic non-malignant conditions. This paper will discuss the clinical case of an adolescent with complex regional pain syndrome (CRPS) and also use this case to highlight the clinical decision and ethical dilemmas faced by the healthcare team.

\section{CASE SUMMARY}

A 15 year old girl presented with chronic left leg pain for several months. The pain was shooting in nature which radiated to her left foot and was associated with hyperpathia. She was unable to sleep most nights due to the severity of the pain. She denied any trauma to her back. She also denied any psychological trauma that has led to her current state. On clinical examination, her peripheral and central nervous system examination were both normal. She had no spinal lesions but her range of movement of her left lower limb was restricted due to her pain. Further investigations including tumour markers, imaging of her legs and radioisotope scan were negative. With the absence of positive findings on her investigations and the inability to locate an organic cause for the pain, she was diagnosed with CRPS. Several pharmacological options were used to manage the pain by the palliative care team and the acute pain services, but her pain persisted. She was hospitalised for several weeks. The key aims of this admission were to use multi-disciplinary team management to achieve symptom control, monitoring of clinical symptoms, and educating the patient and family about CRPS.

\section{ISSUES}

CRPS is a disorder characterised by pain with sympathetic nervous system involvement. It is comprised of various diagnostic criteria such as continuing pain which is disproportionate, and it is associated with vasomotor, sensory, or motor changes (2). The difficulties associated with CRPS lie in the fact that no organic cause for the pain is discernible. It is important to eliminate serious causes of pain, such as tumours and neuropathic pain as well as assess the psychological aspects of pain. Scoring pain severity can be challenging, as no objective tools exist for children. Though there are verbal and visual pain severity scales, these can be unreliable and subject to interpretation. Management of CRPS requires the clinician to engage in discussion with the patient to understand the debilitating nature of this condition and to acknowledge that their pain is real, in spite of the lack of underlying organic causes for the pain. It is also important to provide psychological support and ensure that somatic disorder is excluded.

Specialised centres which manage CRPS are scarce. In the absence of specialised units, collaboration between hospital and community-based health professionals is vital to ensure that the patient is well supported and facilitate normalisation of mobility and functionality. Treatment should be focused on restoring functional capacity by the multidisciplinary team. This involves the gradual introduction of movement and 
desensitisation to the sensory stimulus to reset the central processing in the nervous system (2). If improvement is poor, interventions should be aimed at patients' comfort and confidence. Strategies such as psychotherapy, cognitive behavioural therapy and pharmacological agents can be used if indicated. In our case, medications were given in the early stage due to uncertainty of the diagnosis. She developed uncontrolled symptoms which warranted maximising drug therapy but this was minimally successful especially in pain control. Once the patient's diagnosis of CRPS became clearer, directed and rationalised treatment was easier to deliver.

The management of CRPS can be challenging for several reasons. Firstly, assessment of pain severity is different for acute and chronic pain conditions. Determination of severity or emergency relief can be difficult due to differences of personal experiences and reporting scale manipulation related to pain. Chronic pain scale is subjected to scrutiny and not validated in children. Secondly, patients may be mistakenly labelled as having a psychological issue or somatisation which can compromise rapport and success of the therapy. It is important for health care personnel to show empathy and reserve judgement regarding the intention to treatment due to psychological domain. The multidisciplinary team perspective is critical to ensure that clinical, emotional, psychosocial and spiritual components are equally addressed. Allied health professional referrals may be warranted is medical measures fail. Involvement of an occupational therapist and a physiotherapist aimed at achieving functional restoration in synchrony with pharmacological treatment is vital in alleviating pain and restoring functionality. It is vital to synchronise these parallel approaches to achieve the best solution and outcomes for the patient. CRPS patients will also need psychological support, another treatment modality, to address issues related to anxiety and depression.

\section{ETHICAL ISSUE DISCUSSION}

The ethical approach for treatment in this case of non-malignant chronic pain in which symptoms are uncontrolled symptoms, is to focus on alleviating the pain. This can be achieved by outlining if clear treatment objectives. In palliative care, the principle focus is that of symptom control, which can be facilitated by using pharmacological agents. By using the WHO pain ladder, health professionals can rationalise pain management. Subsequent information and investigations revealed the absence of either mechanical or neoplastic origin would demand less aggressive treatment course. Best interest of the patient at any course of treatment changed with available evidence and knowledge accordingly. The approach for psychological and perhaps psychiatric ground may pose different avenue of care. The best interest for the patient shifts as the remedy of care is based on sound evidence from investigations performed or having an accurate diagnosis of the underlying medical problems at that particular time of care.

Another aspect of ethical value is to ensure treatment given does benefit the patient. The use of opioid is thought to cause side effects, particularly in end of life, such as respiratory depression. But the use of principle of double effect has been associated with the use of opioid at terminal stage (3). In contrast our case depicts a different conundrum, where primarily the use of early opioid mainly to ease pain. Complexity of in managing pain stimulus can be a challenging task. Various known factors which modify pain include personal experience, type of pain i.e. neuropathic or nociceptive and presence of other component such as psychological issue. Detail pain history is required to elicit or exclude serious underlying problem. Patient also has autonomy to decision process. In adolescent age beyond 13, the premise is to get them on board to understand the illness. Although most decision should be run through with caregivers but patient has the right to make a joint decision in the care. The knowledge should include 
the disease process, treatment modality, choice of treatment, side effects and long term sequalae. Applying 'Gillick' competence would be a helpful interface not only allowing share decision process from health care and patient with family members. If parental decision takes over the patient's request, further friction may lead to unsuccessful care and friction in relationship. It's vital that all involved understand the treatment goals.

The management of complex symptomatology especially in noncancer patient require much of a broader approach. This would involve many medical disciplines. This scenario has similar applicability to end of life where total pain can be investigated into different lens. Psychological, social, emotional, financial and spiritual elements must be explored apart from physical component. In our case, the medical perhaps is supposed to be geared as last resort. The nature of this patient's illness requires a focus management from rehabilitative perspective. Involvement of allied healthcare professionals, such as occupational therapist, physiotherapists as well as psychologists, have allowed recovery and rehabilitation process. Similar application can be universally applied in other symptoms management i.e. dyspnoea, or fever or lethargy. Goals would always be focused toward functionality and gradual process should be initiated. Many other personnel like music therapist, spiritual healer may be valuable to distract patients from focusing on disease. For children, considering them to get involved in schooling as part of routine activity, this will allow them to have normality and control of their lives. Peer support and recognition would be vital for adolescent wants to get back on track. Element of bullying, low self-esteem or fear should be dealt swiftly through professionals help.

Treatment of choice must also be justifiable based on the underlying diagnosis, age maturity and financial cover. Various options can be table, but often individual plan should be recognised based on personal resources availability. But, in the event where resources are minimal, man power is limited, the focus of treatment must be matched locally. If this is not feasible, then referral to a specialised centre is warranted. Unfortunately, in many circumstances, the service or support is scarce. The service is structurally expensive and difficult to enrol in. Ethically, if best interest is not served, second best interest may be the next option. Regimented, expensive, or better treatment may not be readily available, thus using a self-assembled team can be the first step around. This is also can be applied to tackle different symptomatology by adhering to the basic needs and finding the root cause. The modality of treatment can go beyond the scope of medicine. The use of drug as a modality of combating symptom should be minimised and reserved in difficult circumstances. It is beyond the scope of this writing to discuss potential issue related to drug usage, as basic tenet again should be measurable from the principle of justice, beneficence and non-maleficence. Discussion on this final step requires a more in depth discussion after all steps known have failed. Even if drug is used, it should be to assist, not to eradicate the total pain because of the nature of the condition.

Somatisation or fabricated induced illness is another difficult clinical enigma. Patient may well fabricate the symptom to achieve her own personal intention. It may also manifest as psychological distress, or anxiety related symptoms which in turn leading exaggerated medical intervention by healthcare personnel. Somatisation will always become a differential. One has to be certain in diagnosis to prevent any unwanted outcome. Psychiatric assessment will give more accurate details on the issue related to psychological intent. Uncertainty to establish diagnosis has delayed proper management in this group. If somatisation or fabricated induced illness is suspected, the ethical thing to do is to stop exploring or inventing treatment. Negotiation on what be achieved can be done, and adolescent 
past trauma may be discussed to ensure psychological component is addressed.

Would end of life strategy be better used in this scenario? There is part where palliative care team may be useful - Communication and use of pain relief. Most of treatment strategy like symptom relief does not oppose the main principle of treatment. We involve palliative care team despite unrelated to end of life or terminal cases. Ultimately, meaningful intervention would integrate effective communication with pain management strategy. Palliative care plays important role especially in the early trajectory of the illness, by building rapport, gradual approach on intervention following the trajectory of the disease. Palliative should be well versed with pain management in either, oncology or non-oncology cases, even cure is imminent. The concept of referral to palliative should be broaden not focused on definite end of life cases only but to other spectrum of difficult uncontrolled symptom management $(4,5)$.

\section{CONCLUSION}

Management of uncontrolled symptoms such as pain, illustrated in this case, may well trigger many aspects of ethical discussion following the diagnosis. In an attempt to help patients, one cannot be judgemental to label the patient with psychological or psychiatric illness, but to acknowledge the illness by showing empathy and be balance on the care and treatment. The course of treatment may largely be influenced by significant impression on underlying illness. Ethically, the intervention must be based on sound ethical principles, moral values and agreed decision from family and adolescent, despite the limitation on consensual age limit and competency. Successful treatment would be achieved through cooperation between the assigned team efforts towards achieving the treatment goals.

\section{REFERENCES}

1. Sullivan M, Ferrell B. Ethical challenges in the management of chronic nonmalignant pain: negotiating through the cloud of doubt. The Jour of Pain. 2005;6:2-9. https://doi. org/10.1016/j.jpain.2004.10.006.

2. Harden RN. Complex regional pain syndrome. Br J Anaesth. 2001;87:99-106. https://doi.org/10.1093/bja/87.1.99.

3. Sykes N, Thorns A. The use of opioid and sedatives at the end of life. Lancet Oncol. 2003;4:312-16. https://doi.org/10.1016/S14 70-2045(03)01079-9.

4. Ellershaw J, Ward C. Care of dying patient: the last hours or days of life. BMJ. 2003;326:30-4. https://doi.org/10.1136/bmj. 326.7379 .30 .

5. Sykes N, Thorns A. Sedative use in the last week of life and the implications for end of life decision making. Arch Intern Med. 2003;163:341-4. https://doi.org/10.1001/ archinte.163.3.341. 